\title{
Joint effect of platelet distribution width and stent surface area on major adverse cardiovascular events after percutaneous coronary intervention
}

\author{
Xiaofeng Zhang ${ }^{1,2 \#}$, Haiyan Ge ${ }^{3 \#}$, Xiaoxuan Gong ${ }^{1}$, Jing Wang ${ }^{1}$, Chunjian Li $^{1}$ \\ ${ }^{1}$ Department of Cardiology First Affiliated Hospital of Nanjing Medical University, Nanjing, China; ${ }^{2}$ The Second Hospital of Nanjing, Nanjing \\ University of Chinese Medicine, Nanjing, China; ${ }^{3}$ Department of Ultrasound Zhongshan Rehabilitation Hospital of Jiangsu Province, Nanjing, \\ China \\ Contributions: (I) Conception and design: C Li; (II) Administrative support: X Zhang, C Li; (III) Provision of study materials or patients: X Zhang, \\ H Ge, X Gong; (IV) Collection and assembly of data: X Zhang, H Ge, X Gong; (V) Data analysis and interpretation: X Zhang, J Wang; (VI) \\ Manuscript writing: All authors; (VII) Final approval of manuscript: All authors. \\ "These authors contributed equally to this work. \\ Correspondence to: Chunjian Li. Department of Cardiology First Affiliated Hospital of Nanjing Medical University, Nanjing, China. \\ Email: lijay@njmu.edu.cn.
}

Background: This study was conducted to analyze the influences of stent surface area (SSA), platelet distribution width (PDW), and the joint effect of these 2 risk factors on major adverse cardiovascular events (MACEs) in patients treated with percutaneous coronary intervention (PCI) together with drug-eluting stent (DES) implantation.

Methods: Based on a cross-sectional survey conducted between 2011 and 2012, a prospective cohort study was enrolled consiting of 442 patients who had undergone PCI with DES implantation. We categorized the participants into 4 subgroups according to PDW and SSA. Cox proportional hazards models were applied to explore the correlation of PDW and SSA with MACE incidence.

Results: During the 12 months of follow-up time, 87 patients experienced MACEs, which included 4 deaths (4.6\%), 5 nonfatal myocardial infarctions (MIs) (5.75\%), 9 ischemic strokes (10.34\%), and 73 clinically relevant bleeding episodes (83.91\%). The risks of MACEs were decreased by SSA and increased by PDW. However, the association of PDW or SSA with MACE was not statistically significant. Compared with the patients with $\mathrm{PDW} \geq 13.5 \%$ and SSA $<358.14 \mathrm{~mm}^{2}$, the multivariable adjusted hazard ratios [HRs; 95\% confience interval (CI)] of the total MACEs for the patients with PDW $<13.5 \%$ and SSA $\geq 358.14 \mathrm{~mm}^{2}$, and with $\mathrm{PDW} \geq 13.5 \%$ and SSA $\geq 358.14 \mathrm{~mm}^{2}$ were 0.94 (95\% CI: $0.55-1.64$ ) and 0.37 (95\% CI: 0.18-0.76), respectively. Additionally, the patients in the group of $\mathrm{PDW}<13.5 \%$ and SSA $<358.14 \mathrm{~mm}^{2}$, and PDW $\geq 13.5 \%$ and SSA $\geq 358.14 \mathrm{~mm}^{2}$ had respective HRs of 0.47 (95\% CI: $0.24-0.91$ ) and 0.28 (95\% CI: 0.13-0.63) for 12 -month bleeding events when PDW $\geq 13.5 \%$ and SSA $<358.14 \mathrm{~mm}^{2}$ was used as a group reference.

Conclusions: Our present results suggest that the joint effect of PDW and SSA was significantly correlated to MACE development in the patients treated with PCI (with DES implantation).

Keywords: Stent surface area(SSA); platelet distribution width (PDW); percutaneous coronary intervention (PCI); major adverse cardiovascular events (MACEs)

Submitted Apr 02, 2021. Accepted for publication May 21, 2021.

doi: 10.21037/apm-21-1088

View this article at: http://dx.doi.org/10.21037/apm-21-1088

(c) Annals of Palliative Medicine. All rights reserved. 


\section{Introduction}

Coronary artery disease (CAD) accounts for more than 610,000 deaths annually worldwide and is a leading cause of global morbidity and mortality (1). With the development of percutaneous coronary intervention (PCI) over the past decades, substantial breakthroughs have been made in the treatment of CAD $(2,3)$. Moreover, the incidence of major adverse cardiovascular events (MACEs), including cardiovascular death, nonfatal myocardial infarction (MI), and ischemic stroke, have been further reduced in the patients since the second-generation drug-eluting stent (DES) was introduced into clinical practice (4-6). However, some patients still show poor prognosis after DES implantation.

Being an index reflecting heterogeneous platelet size, platelet distribution width (PDW), plays an important role in the development of atherothrombosis and atherosclerotic plaque rupture (7-9). In a Polish study, Kern et al. reported that PDW is a low-cost and reliable parameter forthe 1-year MACE rate after PCI within coronary bifurcation lesions (10). In addition, several stent characteristics, such as stent length and diameter, have also been reported as important parameters for the subsequent risk of MACEs after PCI procedures (10]. For instance, Plitt et al. reported that a stent diameter (SD) value of $3.25-3.5 \mathrm{~mm}$ and and anSD value $>3.5 \mathrm{~mm}$ were significantly associated with a $21 \%$ and $34 \%$ lower risk for MACE rates, respectively, in comparision with an SD level of $\leq 2.5 \mathrm{~mm}$ (11). Stent surface area (SSA) is a comprehensive index value that can reflect the length and diameter of stent. However, the effect of SSA on the risk of adverse outcomes after PCI remains unclear. Therefore, the purpose of our research was to explore SSA, PDW, and the combined effect of SSA and PDW on the occurrence of MACEs in patients who undergo PCI with DES implantation. We present the following article in accordance with the STROBE reporting checklist (available at http://dx.doi.org/10.21037/apm-21-1088).

\section{Methods}

\section{Research design and patients characteristics}

This study was conducted in the First Affiliated Hospital of Nanjing Medical University, Nanjing, China (12). The study protocol received approval from the Ethics Committee of the First Affiliated Hospital of Nanjing Medical University (No. 2011036) and conformed to the ethical principles of the Declaration of Helsinki (as revised in 2013). Briefly, between January 2011 and December 2012, patients with acute coronary syndrome (ACS) or stable CAD with coronary stent implantation were consecutively screened for the present study. Patients were eligible for enrollment (I) if they were $\geq 18$ yearsof age, and (II) if they had undergone PCI with second-generation DES implantation for CAD. The exclusion criteria were the following: (I) patients had previously undergone coronary artery bypass surgery; and (II) patients suffered from debilitating conditions, including advanced malignancies, severe liver and kidney dysfunctions, severe autoimmune diseases,or cerebrovascular accidents with major sequelae. Finally, 442 patients were recruited in the present analysis. The written informed consents were asked from each patient before their enrollment in the study.

\section{Data collection, follow-up, and sample measurements}

The demographic characteristics, and medical and social histories were collected from each included patient. The synergy between PCI with taxus and cardiac surgery (SYNTAX) score, a validated scoring system for the complexity of coronary lesions, was prospectively calculated (using the online tool accessed at http://www.syntaxscore. com/calculator/start.htm) by 3 experienced investigators. In the present study, body mass index (BMI) was calculated as the weight in kilogramsdevided by the square of the height in meters. We measured the blood pressure (BP) with an Omron HEM-907 sphygmomanometer in sitting position after 5 minutes rest (Omron Corp., Tokyo, Japan). SSA was calculated using the following formula: $\mathrm{SSA}=\pi \times \mathrm{SD}$ (stent diameter) $\times$ SL (stent length). Triglycerides (TG), high-density lipoprotein cholesterol (HDL-c), low-density lipoprotein cholesterol (LDL-c), fasting plasma glucose (FPG), and (TC) were detected using commercial reagents on a chemistry analyzer (AU5400 Analyzer, Beckman Coulter, CA, USA).

We defined thesuccess of PCI according to following criteria determined by consensus of the international experts in our field: (I) a residual diameter stenosis $>25 \%$ and (II) obtainingan enhanced figure without further delay to the distal coronary artery (thrombolysis in MI3 flow) in patients' selected lesions. After the PCI procedure, all patients received aspirin $81-100 \mathrm{mg} \mathrm{qd}$, and clopidogrel $75 \mathrm{mg}$ qd or ticagroler $90 \mathrm{mg}$ bid. Clinical follow-up was arranged at 30 days, 3 months, 6 months, and 12 months by inviting patients or their relatives to finish a standardized questionnaire. The participants who dropped out of follow- 
up were excluded from the survival analysis of the study.

\section{Primary endpoint}

The primary endpoint of MACEs included all-cause death, nonfatalMI, ischemic stroke, and clinically relevant bleeding within 1-year after PCI. We defined all-cause deathas any death during or after the PCI procedure thatwas considered to be of cardiac origin unless a diagnosis of anoncardiaccause could be assessed. MIwas defined as an ischemic symptom arising within 3 months before enrollment with new electrocardiographic changes and increased concentration of circulatingcardiac troponin $(\geq 0.5 \mu \mathrm{g} / \mathrm{L})$. Ischemic stroke events were determined according to the Bleeding Academic Research Consortium classification (13). In addition, clinically relevant bleeding was defined as bleeding meeting any of the major criteria according to GUSTO (global utilization of streptokinase and TPA for occluded arteries) or ACUITY (acute catheterization and urgent intervention triage strategy) scales, as well as any other types of bleeding that required medical care after hospital discharge.

\section{Statistical analysis}

According to the PDW and SSA, we divided patients into 4 subgroups: $\mathrm{PDW} \geq 13.5 \%$ with $\mathrm{SSA}<358.14 \mathrm{~mm}^{2}$, PDW $<13.5 \%$ with SSA $<358.14 \mathrm{~mm}^{2}, \mathrm{PDW} \geq 13.5 \%$ with SSA $\geq 358.14 \mathrm{~mm}^{2}$, and PDW $<13.5 \%$ with SSA $\geq 358.14 \mathrm{~mm}^{2}$. For the comparison ofbaseline characteristics, continuous variables are displayed as mean \pm standard deviation if the sample was normally distributed, or as median with $25^{\text {th }}$ and $75^{\text {th }}$ percentilesif it was skewed.Categorical variables are shownas numbers or percentages. The $\chi^{2}$ test or Fisher's exact test was used to compare the characteristics of participants,. We used log-rank tests to adjustthe cumulative risk of outcomes among the 4 subgroups. For the purpose of testingjoint effects of PDW and SSA on the clinical outcomes after PCI, we used multivariate Cox proportional hazard models for calculation of the hazard ratios (HRs) with $95 \%$ confidence intervals (CIs) of MACE across the 4 subgroups, adjusting for potential confounders of age, sex, current smoking habits, drinking habits, BMI, hypertension, diabetes mellitus (DM), and SYNTAX score. Statistical analysis wasperformedwith R 3.4.0 (Vienna, Austria) and SAS version 9.1 (Cary, NC, USA). All $\mathrm{P}$ values $<0.05$ (2-tailed) weredeemed statistically significant.

\section{Results}

During the follow-up period, 87 patients experienced MACEs, which included 4 deaths (4.6\%), 5 nonfatal MIs (5.75\%), 9 ischemic strokes (10.34\%), and 73 clinically relevant bleeding episodes (83.91\%). Patients' baseline clinical and demographic characteristics are summarized according to PDW and SSAin Table 1 The patients with PDW $<13.5 \%$ either in the SSA $<358.14 \mathrm{~mm}^{2}$ group or in the SSA $\geq 358.14 \mathrm{~mm}^{2}$ group tended to have higher amounts of TC, HDL-c, and LDL-c. Moreover, patients with SSA $\geq 358.14 \mathrm{~mm}^{2}$ either in the PDW $<13.5 \%$ group or in the PDW $\geq 13.5 \%$ group tended to have higher SYNTAX score, longer stent length, and larger SSA.

We tested the effects of PDW and SSA with MACE in the patients after PCI (Table 2). The risks of clinically relevant bleeding were decreased by SSA and increased by PDW. However, HRs of MACEs for PDW $\geq 13.5 \%$ and SSA $\geq 358.14 \mathrm{~mm}^{2}$ were respectively 0.94 (95\% CI: 0.62 $1.44)$ and 0.86 (95\% CI: $0.55-1.36$ ), after adjustments were made for the possible confounders of age, sex, smoking habits, drinking, BMI, hypertension, DM, and SYNTAX score. Additionally, we found no significant association between PDW or SSA and bleeding events in these patients after PCI procedure,

The cumulative incidence rates of total MACEs among the 4 subgroups were $27.82 \%$ for $\mathrm{PDW} \geq 13.5 \%$ and SSA $<358.14 \mathrm{~mm}^{2}, 26.09 \%$ for PDW $<13.5 \%$ and SSA $\geq 358.14 \mathrm{~mm}^{2}, 17.14 \%$ for PDW $<13.5 \%$ and SSA $<358.14 \mathrm{~mm}^{2}$, and $10.28 \%$ for PDW $\geq 13.5 \%$ and SSA $\geq 358.14 \mathrm{~mm}^{2}$ (log-rank $\left.\mathrm{P}=0.007\right)$. As shown in Table 3, compared with the patients with $\mathrm{PDW} \geq 13.5 \%$ and SSA $<358.14 \mathrm{~mm}^{2}$, the multivariable adjusted HRs of total MACEs for the patients with PDW $<13.5 \%$ and SSA $\geq 358.14 \mathrm{~mm}^{2}$ were 0.94 (95\% CI: $0.55-1.64$ ). The patients with $\mathrm{PDW} \geq 13.5 \%$ and $\mathrm{SSA} \geq 358.14 \mathrm{~mm}^{2}$ were at the lowest risk of total MACEs during 12-month follow-up (HR $=0.37,95 \%$ CI: 0.18-0.76, P=0.007).

Because more than $80 \%$ of the MACEs were clinically relevant bleeding events, we further analyzed the combined effect of PDW and SSA on the risk of bleeding in this follow-up study. The cumulative incidence rates of bleeding among the 4 subgroups were $24.35 \%$ for PDW $\geq 13.5 \%$ and SSA $<358.14 \mathrm{~mm}^{2}, 20.00 \%$ for PDW $<13.5 \%$ and SSA $\geq 358.14 \mathrm{~mm}^{2}, 13.33 \%$ for PDW $<13.5 \%$ and SSA $<358.14 \mathrm{~mm}^{2}$, and $7.48 \%$ for $\mathrm{PDW} \geq 13.5 \%$ and SSA $\geq 358.14 \mathrm{~mm}^{2}(\log$-rank $\mathrm{P}=0.004)$. Compared with those of patients with $\mathrm{PDW} \geq 13.5 \%$ and SSA $<358.14 \mathrm{~mm}^{2}$, the 
Table 1 Patients characteristics in various PDW and SSA subgroups

\begin{tabular}{|c|c|c|c|c|c|}
\hline Variables & $\begin{array}{l}\text { PDW } \geq 13.5 \% \text { with } \\
\text { SSA }<358.14 \mathrm{~mm}^{2}\end{array}$ & $\begin{array}{l}\text { PDW }<13.5 \% \text { with } \\
\text { SSA }<358.14 \mathrm{~mm}^{2}\end{array}$ & $\begin{array}{l}\text { PDW } \geq 13.5 \% \text { with } \\
S S A \geq 358.14 \mathrm{~mm}^{2}\end{array}$ & $\begin{array}{l}\text { PDW }<13.5 \% \text { with } \\
\text { SSA } \geq 358.14 \mathrm{~mm}^{2}\end{array}$ & $P$ value \\
\hline Number of participants & 115 & 105 & 107 & 115 & \\
\hline Age (years) & $65.01 \pm 11.13$ & $63.39 \pm 10.95$ & $64.40 \pm 10.42$ & $63.91 \pm 10.59$ & 0.718 \\
\hline Male (n, \%) & $87(75.65)$ & $81(77.14)$ & $84(77.78)$ & 87 (75.65) & 0.975 \\
\hline Drinking (n, \%) & $31(26.96)$ & $27(25.71)$ & $26(24.07)$ & 27 (23.48) & 0.929 \\
\hline BMI $\left(\mathrm{kg} / \mathrm{m}^{2}\right)$ & $24.70 \pm 3.13$ & $25.11 \pm 2.83$ & $25.14 \pm 3.00$ & $24.92 \pm 2.86$ & 0.675 \\
\hline TC (mmol/L) & $4.02 \pm 0.92$ & $4.51 \pm 1.25$ & $4.37 \pm 1.14$ & $4.40 \pm 1.53$ & 0.020 \\
\hline TG (mmol/L) & $1.62 \pm 1.23$ & $1.63 \pm 1.00$ & $1.78 \pm 1.06$ & $1.87 \pm 2.36$ & 0.550 \\
\hline SYNTAX score & $12.41 \pm 7.26$ & $14.36 \pm 9.11$ & $18.86 \pm 7.82$ & $19.77 \pm 8.05$ & $<0.001$ \\
\hline Hypertension (n, \%) & $81(70.43)$ & $67(63.81)$ & 77 (71.30) & $82(71.30)$ & 0.577 \\
\hline Diabetes mellitus (n, \%) & $37(32.17)$ & $31(29.52)$ & $29(26.85)$ & $32(27.83)$ & 0.828 \\
\hline Stent length (mm) & $24.51 \pm 6.03$ & $24.15 \pm 7.29$ & $66.39 \pm 25.63$ & $64.71 \pm 23.17$ & $<0.001$ \\
\hline Stent diameter (mm) & $3.01 \pm 0.43$ & $3.01 \pm 0.49$ & $3.03 \pm 0.36$ & $3.00 \pm 0.30$ & 0.951 \\
\hline Stent surface area $\left(\mathrm{mm}^{2}\right)$ & $230.70 \pm 58.54$ & $229.11 \pm 69.21$ & $622.86 \pm 232.40$ & $608.75 \pm 224.86$ & $<0.001$ \\
\hline \multicolumn{6}{|l|}{ Outcome (n, \%) } \\
\hline Total MACE & 32 (27.82) & $18(17.14)$ & $11(10.28)$ & 30 (26.09) & 0.007 \\
\hline
\end{tabular}

BMI, body mass index; TC, total cholesterol; TG, triglycerides; HDL-c, high density lipoprotein-cholesterol; LDL-c, low density lipoproteincholesterol; FPG, fast plasma glucose; MACE, major adverse cardiovascular events.

multivariable adjusted HRs of bleeding for the patients with $\mathrm{PDW}<13.5 \%$ and SSA $<358.14 \mathrm{~mm}^{2}$, and PDW $<13.5 \%$ and SSA $\geq 358.14 \mathrm{~mm}^{2}$ were 0.47 (95\% CI: 0.24 $0.91, \mathrm{P}=0.026$ ) and 0.80 (95\% CI: $0.44-1.45, \mathrm{P}=0.457$ ), respectively. The patients with $\mathrm{PDW} \geq 13.5 \%$ and SSA $\geq 358.14 \mathrm{~mm}^{2}$ were at the lowest risk of clinically relevant bleeding ( $\mathrm{HR}=0.28,95 \% \mathrm{CI}$ : 0.13-0.63, $\mathrm{P}=0.002$; Table 4). However, other than clinically relevant bleeding, no significant combined effects were detected between PDW and SSA on the development of MACEs in the patients who underwent PCI with DES implantation.

\section{Discussion}

Although the data on the association between SSA and MACEs after PCI is very limited, the length and diameter of stent have been reported as independent predictors of MACEs after PCI with DES implantation (11,14-17). For instance, the results from a meta-analysis found that females with smaller SDstreated with PCI had ahigher risk of definite stent thrombosis and target lesion revascularization, consistent with earlyand newgenerations of DES (18). However, in a prospective study, Adnan et al. (19) 
Table 2 Associations of PDW and SSA with MACEs and bleeding adverse events in patients treated with PCI with DES

\begin{tabular}{|c|c|c|c|}
\hline Variables & \multicolumn{3}{|c|}{ Multivariable adjusted $^{\mathrm{a}}$} \\
\hline \multicolumn{4}{|l|}{ MACE } \\
\hline PDW (per 1\%) & 0.97 & $0.90-1.05$ & 0.426 \\
\hline$P D W \geq 13.5 \%$ & 0.94 & $0.62-1.44$ & 0.786 \\
\hline $\mathrm{SSA} \geq 358.14 \mathrm{~mm}^{2}$ & 0.86 & $0.55-1.36$ & 0.519 \\
\hline \multicolumn{4}{|l|}{ Bleeding } \\
\hline PDW (per 1\%) & 0.97 & $0.89-1.05$ & 0.432 \\
\hline$P D W \geq 13.5 \%$ & 0.99 & $0.62-1.58$ & 0.963 \\
\hline
\end{tabular}

a, adjusted for potential coufounders: age, sex, smoking habits, drinking haibits, BMI, hypertension, diabetes mellitus, and SYNTAX score. PDW, platelet distribution width; SSA, stent surface area; MACEs, major adverse cardiovascular events; PCI, percutaneous coronary intervention; DES, drug-eluting stent; $\mathrm{Cl}$, confidence interval; HR, hazard ratio.

Table 3 Joint effects of PDW and SSA on MACEs in the patients treated with PCI with DES

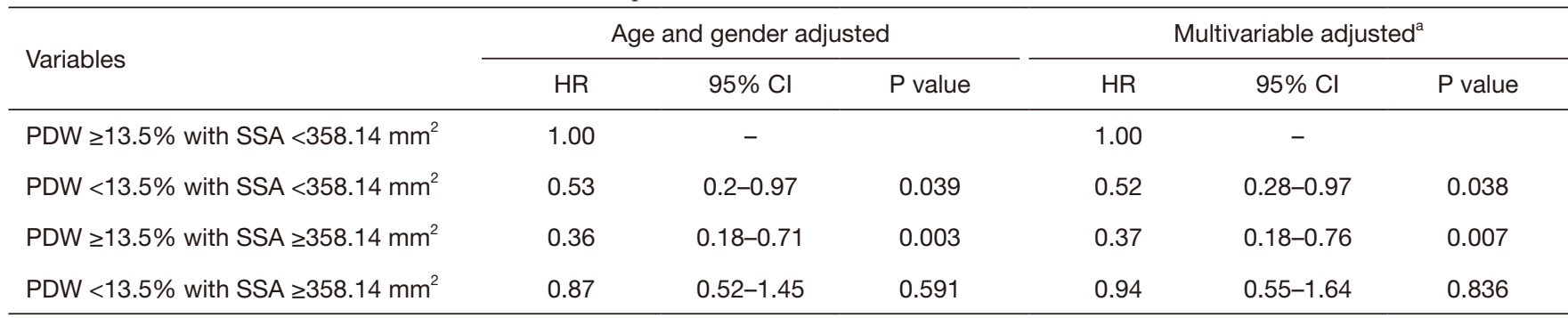

a, adjusted for potential coufounders: age, sex, smoking habits, drinking, BMI, hypertension, diabetes mellitus, and SYNTAX score. PDW, platelet distribution width; SSA, stent surface area; MACEs, major adverse cardiovascular events; PCI, percutaneous coronary intervention; DES, drug-eluting stent; $\mathrm{Cl}$, confidence interval; HR, hazard ratio.

Table 4 Joint effects of PDW and SSA on bleeding events in the patients treated with PCI with DES

\begin{tabular}{|c|c|c|c|c|c|c|}
\hline Variables & \multicolumn{3}{|c|}{ Age and gender adjusted } & \multicolumn{3}{|c|}{ Multivariable adjusted $^{a}$} \\
\hline PDW $\geq 13.5 \%$ with SSA $<358.14 \mathrm{~mm}^{2}$ & 1.00 & - & & 1.00 & - & \\
\hline PDW $<13.5 \%$ with SSA $<358.14 \mathrm{~mm}^{2}$ & 0.47 & $0.24-0.91$ & 0.025 & 0.47 & $0.24-0.91$ & 0.026 \\
\hline$P D W \geq 13.5 \%$ with $S S A \geq 358.14 \mathrm{~mm}^{2}$ & 0.29 & $0.13-0.63$ & 0.002 & 0.28 & $0.13-0.63$ & 0.002 \\
\hline
\end{tabular}

a, adjusted for for potential coufounders: parameters including age, sex, smoking habits, drinking habits, BMI, hypertension, diabetes mellitus, and SYNTAX score. PDW, platelet distribution width; SSA, stent surface area; PCI, percutaneous coronary intervention; DES, drug-eluting stent; $\mathrm{Cl}$, confidence interval; HR, hazard ratio. 
reported that the length and diameter of stent did not have any impact on the short-term clinical outcomes of DES inpatients in stable CAD status.In addition, results from another meta-analysis observed mean stent length was longer in those patients suffering stent thrombosis, indicating that the risk of stent thrombosis after DES implantation is related to stent length (20). Although we observed that MACE incidence was increased with SSA, no statistically significant association between SSA and the risk of MACE development was detected in this study. Considering that the patients receiving the stent with larger SSA had a decresedprevalance of MACEs during a followup period of 12 months, more trials should be made to study the influence of SSA on the clinical outcomes in the patients after the PCI procedure.

PDW is generally used to measure fractions of enzymatically and metabolically more active, and larger platelets. PDWrefers to the variability in the size of platelet, which is enlargedwhen plateletsbecome activated $(21,22)$. PDW is regarded as a more specific marker of platelet reactivity,as it is stable and notaffected by the distention of a single swollen platelet $(23,24)$. However, the associations between PDW and MACEs in the patients undergoing PCI have not been fully understood. For example, Celik et al. (25) found that PDW is an independent parameter of no reflow and in-hospital MACEs in ST-elevation myocardial infarction (STEMI) patients undergoing PCI. In addition, a Polish study found that PDW with a cutoff value of $15.8 \%$ could predict the risk of MACEs with $79 \%$ sensitivity and $47 \%$ specificity (9). However, Verdoia et al. (26) reported that PDW did not addto the risk of periprocedural MI, and thus suggested it should not be regardedasa risk factor of thrombotic periprocedural complications in patients after PCI. In our analysis, we observed that PDW did not have any influence on the risks of total MACEsor clinically relevant bleeding events. One possible explanation for this finding is theshorter follow-upperiodof our research (most patients were followed for a period of just over 1 year). Thus, the long-term effects of PDW on the clinical outcomes after DES implantation should be more extensively analyzed in the future.

Our study was the first to investigate the joint effects of PDW and SSA on MACEs in the patients who have undergone PCI. After making adjustments for the potential confounders, among the patients with $\mathrm{PDW} \geq 13.5 \%$, we observed that the patients with SSA $<358.14 \mathrm{~mm}^{2}$ had an associated 2.70- and 3.57-fold increased risk for MACEs and bleeding compared with the patients with SSA $\geq 358.14 \mathrm{~mm}^{2}$, respectively. The results indicate that stents with a larger SSA might have an additional preventive effect on MACE development in the patients with larger PDW. In addition, among the the patients SSA $<358.14 \mathrm{~mm}^{2}$, we observed that the patients with $\mathrm{PDW} \geq 13.5 \%$ were associated with a 1.92 fold and 2.13-fold increased risk for MACEs and bleeding compared with the patients with $\mathrm{PDW}<13.5 \%$, respectively. It suggested that the smaller PDW could prevent MACE occurrence in patients undergoing PCI with stents of larger SSA. However, the combined effect of smaller PDW $(<13.5 \%)$ and larger SSA $\left(\geq 358.14 \mathrm{~mm}^{2}\right)$ did not have any influence on MACE development when compared to parameters of $\mathrm{PDW} \geq 13.5 \%$ and SSA $<358.14 \mathrm{~mm}^{2}$. In order toobtain solid and consistent results, future trialsare needed to further confirm our conclusion of the joint effect of PDW and SSA on MACE development in patients undergoing PCI with DES implantation.

There are several limitations in this study that should be considered. First, as they were derived froma study with a single-center design, our findings might not be generalizable to other populations. In addition, considering that our sample size was relatively small, subanalyses were not performed according to SSA and PDW for specific MACEs. Therefore, caution should be taken when interpreting the results. Second, although SSA is strongly correlated with reference vessel diameter and SD $(11,27)$. However, no information on reference vessel diameter has been reported thus far. Third, although weused random monitoring during enrollment of patients and adjudicatedthe events by reviews of electrocardiogramsto collect the information about MACEs, inaccurate outcomes could not be fully avoided. Furthermore, because data were collectedup to a 12-month follow-up, the information on MACEs past this time were not acquired. Finally, although multivariable Cox regressions were used to analyze the HRs (95\% CIs) of MACEs, due to the observational nature of the study, the possibility of unknown and unmeasured confounding factors remains.

In summary, our results suggest that the joint effect of PDW and SSA was significantly correlated to MACE development in patients undergoing PCIs with DES implantation. Additional longitudinal studies with large study samples are needed tofurther clarify the precise effect of PDW and SSA on the long-term clinical effectsoccurring 
in patients after PCI.

\section{Acknowledgments}

Funding: None.

\section{Footnote}

Reporting Checklist: The authors have completed the STROBE reporting checklist. Available at http://dx.doi. org/10.21037/apm-21-1088

Data Sharing Statement: Available at http://dx.doi. org/10.21037/apm-21-1088

Conflicts of Interest: All authors have completed the ICMJE uniform disclosure form (available at http://dx.doi. org/10.21037/apm-21-1088). The authors have no conflicts of interest to declare.

Ethical Statement: The authors are accountable for all aspects of the work in ensuring that questions related to the accuracy or integrity of any part of the work are appropriately investigated and resolved. The study protocol received approval from the Ethics Committee of the First Affiliated Hospital of Nanjing Medical University (No. 2011036) and conformed to the ethical principles of the Declaration of Helsinki (as revised in 2013). The written informed consents were asked from each patient before their enrollment in the study.

Open Access Statement: This is an Open Access article distributed in accordance with the Creative Commons Attribution-NonCommercial-NoDerivs 4.0 International License (CC BY-NC-ND 4.0), which permits the noncommercial replication and distribution of the article with the strict proviso that no changes or edits are made and the original work is properly cited (including links to both the formal publication through the relevant DOI and the license). See: https://creativecommons.org/licenses/by-nc-nd/4.0/.

\section{References}

1. Mack M, Gopal A. Epidemiology, Traditional and Novel Risk Factors in Coronary Artery Disease. Heart Fail Clin 2016;12:1-10.

2. Chacko L, P Howard J, Rajkumar C, et al. Effects of Percutaneous Coronary Intervention on Death and
Myocardial Infarction Stratified by Stable and Unstable

Coronary Artery Disease: A Meta-Analysis of Randomized Controlled Trials. Circ Cardiovasc Qual Outcomes 2020;13:e006363.

3. Al-Lamee RK, Nowbar AN, Francis DP. Percutaneous coronary intervention for stable coronary artery disease. Heart 2019;105:11-9.

4. Cui K, Lyu S, Song X, et al. Drug-eluting balloon versus bare-mental stent and drug-eluting stent for de novo coronary artery disease: A systematic review and metaanalysis of 14 randomized controlled trials. PLoS One 2017;12:e0176365.

5. Lin CF, Chang $\mathrm{YH}, \mathrm{Su} \mathrm{CH}$, et al. Risk of new-onset atrial fibrillation after drug-eluting stent implantation in patients with stable coronary artery disease. Int J Cardiol 2019;291:63-8.

6. Kedhi E, Joesoef KS, McFadden E, et al. Secondgeneration everolimus-eluting and paclitaxel-eluting stents in real-life practice (COMPARE): a randomised trial. Lancet 2010;375:201-9.

7. Temelli B, Yetkin Ay Z, Aksoy F, et al. Platelet indices (mean platelet volume and platelet distribution width) have correlations with periodontal inflamed surface area in coronary artery disease patients: A pilot study. J Periodontol 2018;89:1203-12.

8. Bekler A, Ozkan MT, Tenekecioglu E, et al. Increased Platelet Distribution Width Is Associated With Severity of Coronary Artery Disease in Patients With Acute Coronary Syndrome. Angiology 2015;66:638-43.

9. De Luca G, Venegoni L, Iorio S, et al. Platelet distribution width and the extent of coronary artery disease: results from a large prospective study. Platelets 2010;21:508-14.

10. Kern A, Gil RJ, Bojko K, et al. Platelet distribution width as the prognostic marker in coronary bifurcation treatment. Eur J Clin Invest 2017;47:524-30.

11. Plitt A, Claessen BE, Sartori S, et al. Impact of stent diameter on outcomes following percutaneous coronary intervention with second-generation drug-eluting stents: Results from a large single-center registry. Catheter Cardiovasc Interv 2020;96:558-64.

12. Li J, Fan Y, Zhu T, et al. Clinical pharmacodynamics and long-term efficacy of Talcom vs. Plavix in patients undergoing coronary stent implantation: a randomized study with 5-year follow-up. Eur J Clin Pharmacol 2018;74:1397-403.

13. Kernan WN, Ovbiagele B, Black HR, et al. Guidelines for the prevention of stroke in patients with stroke and transient ischemic attack: a guideline for healthcare 
professionals from the American Heart Association/ American Stroke Association. Stroke 2014;45:2160-236.

14. Konishi H, Miyauchi K, Dohi T, et al. Impact of stent length on clinical outcomes of first-generation and newgeneration drug-eluting stents. Cardiovasc Interv Ther 2016;31:114-21.

15. Yano H, Horinaka S, Ishimitsu T. Impact of everolimuseluting stent length on long-term clinical outcomes of percutaneous coronary intervention. J Cardiol 2018;71:444-51.

16. Redfors B, Chen S, Généreux P, et al. Relationship Between Stent Diameter, Platelet Reactivity, and Thrombotic Events After Percutaneous Coronary Artery Revascularization. Am J Cardiol 2019;124:1363-71.

17. Guttmann OP, Jones DA, Safwan KA, et al. Drug-eluting stents appear superior to bare metal stents for vein-graft PCI in vessels up to a stent diameter of $4 \mathrm{~mm}$. Heart Int 2016;11:e17-e24.

18. Camaj A, Giustino G, Claessen BE, et al. Effect of stent diameter in women undergoing percutaneous coronary intervention with early- and new-generation drug-eluting stents: From the WIN-DES collaboration. Int J Cardiol 2019;287:59-61.

19. Adnan Y, Noor L, Dar MH, et al. Impact of stent length and diameter on short term clinical outcomes of drug eluting stents in patients with stable coronary artery disease. Pak J Med Sci 2017;33:959-62.

20. Moreno R, Fernández C, Hernández R, et al. Drug-eluting stent thrombosis: results from a pooled analysis including 10 randomized studies. J Am Coll Cardiol 2005;45:954-9.
21. Babu E, Basu D. Platelet large cell ratio in the differential diagnosis of abnormal platelet counts. Indian J Pathol Microbiol 2004;47:202-5.

22. Kaito K, Otsubo H, Usui N, et al. Platelet size deviation width, platelet large cell ratio, and mean platelet volume have sufficient sensitivity and specificity in the diagnosis of immune thrombocytopenia. Br J Haematol 2005;128:698-702.

23. Vagdatli E, Gounari E, Lazaridou E, et al. Platelet distribution width: a simple, practical and specific marker of activation of coagulation. Hippokratia 2010;14:28-32.

24. Ihara A, Kawamoto T, Matsumoto K, et al. Relationship between hemostatic factors and the platelet index in patients with ischemic heart disease. Pathophysiol Haemost Thromb 2006;35:388-91.

25. Celik T, Kaya MG, Akpek M, et al. Predictive value of admission platelet volume indices for in-hospital major adverse cardiovascular events in acute ST-segment elevation myocardial infarction. Angiology 2015;66:155-62.

26. Verdoia M, Barbieri L, Schaffer A, et al. Platelet distribution width and the risk of periprocedural myocardial infarction in patients undergoing percutaneous coronary intervention. J Thromb Thrombolysis 2014;37:345-52.

27. Quizhpe AR, Feres F, de Ribamar Costa J Jr, et al. Drugeluting stents vs bare metal stents for the treatment of large coronary vessels. Am Heart J 2007;154:373-8.

(English Language Editor: J. Gray)
Cite this article as: Zhang $\mathrm{X}$, Ge $\mathrm{H}$, Gong $\mathrm{X}$, Wang J, Li C. Joint effect of platelet distribution width and stent surface area on major adverse cardiovascular events after percutaneous coronary intervention. Ann Palliat Med 2021;10(5):5633-5640. doi: 10.21037/apm-21-1088 